\title{
Frequency multiplexed long range swept source optical coherence tomography
}

\author{
Mantas Zurauskas," Adrian Bradu, and Adrian Gh. Podoleanu \\ Applied Optics Group, School of Physical Sciences, University of Kent, CT2 7NH Canterbury, UK \\ *mz63@kent.ac.uk
}

\begin{abstract}
We present a novel swept source optical coherence tomography configuration, equipped with acousto-optic deflectors that can be used to simultaneously acquire multiple B-scans originating from different depths. The sensitivity range of the configuration is evaluated while acquiring five simultaneous B-scans. Then the configuration is employed to demonstrate long range B-scan imaging by combining two simultaneous B-scans from a mouse head sample.
\end{abstract}

(C)2013 Optical Society of America

OCIS codes: (120.3180) Interferometry; (110.4500) Optical coherence tomography; (170.1065) Acousto-optics; (170.0110) Imaging systems; (110.6880) Three-dimensional image acquisition; (140.3600) Lasers, tunable.

\section{References and links}

1. D. Huang, E. A. Swanson, C. P. Lin, J. S. Schuman, W. G. Stinson, W. Chang, M. R. Hee, T. Flotte, K. Gregory, C. A. Puliafito, and J. G. Fujimoto, "Optical coherence tomography," Science 254(5035), 1178-1181 (1991).

2. A. G. Podoleanu, "Optical coherence tomography," J. Microsc. 247(3), 209-219 (2012).

3. W. Wieser, B. R. Biedermann, T. Klein, C. M. Eigenwillig, and R. Huber, "Multi-megahertz OCT: high quality 3D imaging at 20 million A-scans and 4.5 GVoxels per second," Opt. Express 18(14), 14685-14704 (2010).

4. W. Wieser, T. Klein, D. C. Adler, F. Trépanier, C. M. Eigenwillig, S. Karpf, J. M. Schmitt, and R. Huber, "Extended coherence length megahertz FDML and its application for anterior segment imaging," Biomed. Opt. Express 3(10), 2647-2657 (2012).

5. V. Jayaraman, J. Jiang, H. Li, P. Heim, G. Cole, B. Potsaid, J. G. Fujimoto, and A. Cable, "OCT imaging up to $760 \mathrm{Khz}$ axial scan rate using single-mode $1310 \mathrm{~nm}$ MEMs-tunable VCSELs with $>100 \mathrm{~nm}$ tuning range," in in CLEO: 2011-Laser Applications to Photonic Applications, OSA Technical Digest (CD) (Optical Society of America, 2011), paper PDPB2.

6. B. Potsaid, B. Baumann, D. Huang, S. Barry, A. E. Cable, J. S. Schuman, J. S. Duker, and J. G. Fujimoto, "Ultrahigh speed $1050 \mathrm{~nm}$ swept source/Fourier domain OCT retinal and anterior segment imaging at 100,000 to 400,000 axial scans per second," Opt. Express 18(19), 20029-20048 (2010).

7. B. J. Vakoc, D. Fukumura, R. K. Jain, and B. E. Bouma, "Cancer imaging by optical coherence tomography: preclinical progress and clinical potential," Nat. Rev. Cancer 12(5), 363-368 (2012).

8. W. Kang, H. Wang, Y. Pan, M. W. Jenkins, G. A. Isenberg, A. Chak, M. Atkinson, D. Agrawal, Z. Hu, and A. M. Rollins, "Endoscopically guided spectral-domain OCT with double-balloon catheters," Opt. Express 18(16), $17364-17372$ (2010).

9. S. Brand, J. M. Poneros, B. E. Bouma, G. J. Tearney, C. C. Compton, and N. S. Nishioka, "Optical coherence tomography in the gastrointestinal tract," Endoscopy 32(10), 796-803 (2000).

10. A. G. Podoleanu, "Unique interpretation of Talbot Bands and Fourier domain white light interferometry," Opt. Express 15(15), 9867-9876 (2007).

11. B. Hofer, B. Považay, B. Hermann, A. Unterhuber, G. Matz, and W. Drexler, "Dispersion encoded full range frequency domain optical coherence tomography," Opt. Express 17(1), 7-24 (2009).

12. R. A. Leitgeb, C. K. Hitzenberger, A. F. Fercher, and T. Bajraszewski, "Phase-shifting algorithm to achieve highspeed long-depth-range probing by frequency-domain optical coherence tomography," Opt. Lett. 28(22), 22012203 (2003).

13. A. Bradu, L. Neagu, and A. Podoleanu, "Extra long imaging range swept source optical coherence tomography using re-circulation loops," Opt. Express 18(24), 25361-25370 (2010).

14. A. Bachmann, R. Leitgeb, and T. Lasser, "Heterodyne Fourier domain optical coherence tomography for full range probing with high axial resolution," Opt. Express 14(4), 1487-1496 (2006).

15. M. Zurauskas, J. Rogers, and A. G. Podoleanu, "Simultaneous multiple-depths en-face optical coherence tomography using multiple signal excitation of acousto-optic deflectors," Opt. Express 21(2), 1925-1936 (2013).

16. N. A. Riza, "Acousto-optically switched optical delay lines," Opt. Commun. 145(1-6), 15-20 (1998).

17. H. C. Ho, E. H. Young, and W. Seale, "Microwave frequency translation with multiple Bragg cells," Proc. SPIE 1703, 37-42 (1992).

18. A. P. Goutzoulis and D. R. Pape, Design and Fabrication of Acousto-Optic Devices (Dekker, 1994), p. xv, 497 p.

\#185418 - \$15.00 USD Received 19 Feb 2013; revised 30 Apr 2013; accepted 30 Apr 2013; published 2 May 2013 (C) 2013 OSA 1 June 2013 | Vol. 4, No. 6 | DOI:10.1364/BOE.4.000778 | BIOMEDICAL OPTICS EXPRESS 778 
19. G. Liu, Z. Zhi, and R. K. Wang, "Digital focusing of OCT images based on scalar diffraction theory and information entropy," Biomed. Opt. Express 3(11), 2774-2783 (2012).

20. T. Xie, S. Guo, Z. Chen, D. Mukai, and M. Brenner, "GRIN lens rod based probe for endoscopic spectral domain optical coherence tomography with fast dynamic focus tracking," Opt. Express 14(8), 3238-3246 (2006).

21. Z. Ding, H. Ren, Y. Zhao, J. S. Nelson, and Z. Chen, "High-resolution optical coherence tomography over a large depth range with an axicon lens," Opt. Lett. 27(4), 243-245 (2002).

22. L. Liu and N. Chen, "Dynamic focusing with radial gratings for in vivo high resolution imaging," Proc. SPIE 6847, 684718, 684718-8 (2008).

23. W. Wieser, B. R. Biedermann, T. Klein, C. M. Eigenwillig, and R. Huber, "Multi-megahertz OCT: high quality 3D imaging at 20 million A-scans and 4.5 GVoxels per second," Opt. Express 18(14), 14685-14704 (2010).

\section{Introduction}

Optical coherence tomography (OCT) [1] is a non invasive imaging technique that has the potential to provide fast and high resolution depth-resolved information of the scattering properties of biological samples. To obtain an axial scan, time domain (TD)-OCT systems rely on the slow reference arm scanning [2]. Later developed spectral domain (SD)-OCT systems enabled fast acquisition [3] of axial scans within a range of several millimeters. One of the major limitation of the SD-OCT technology remains its depth dependent sensitivity drop, an effect commonly called as the sensitivity "roll-off". In swept source (SS)-OCT systems, the sensitivity roll-off is due to the limited coherence length of the swept source used, while in spectrometer based (SB)-OCT systems, the limited resolution of the spectrometer causes the roll-off [2].

Recent progress in the development of tunable lasers for SS-OCT lead to swept sources with coherence length longer than $1 \mathrm{~cm}[4,5]$. However, when increasing the tuning speed, their coherence length diminishes [6]. There is an interest in imaging objects with axial extension larger than $1 \mathrm{~cm}$ such as the human eye [4], or perform topography of objects with variation in height larger than $1 \mathrm{~cm}$. Therefore, there is a need for optical sources of longer coherence length, or equivalently, of methods that can compensate the sensitivity roll-off with depth.

We recognize that in typical OCT biomedical applications, such as intravascular and gastrointestinal imaging, the light cannot penetrate biological tissue deeper than few millimeters [7] and the loss of backscattered light is a limiting factor, not the roll-off. However, in several biomedical applications such as balloon based endoscopic OCT [8] or gastrointestinal imaging [9], the distance to the sample can vary by few tenths of millimetres and the high imaging range is desired to obtain consistent quality scans.

Various approaches to increase the imaging range have already been reported. Several techniques have been developed to remove the mirror terms [10-12] and effectively double the axial range by giving access to full axial range. A different approach, consisted in using a pair of recirculation loops in the object and reference arms [13] with a differential optical path difference comparable with the axial range given by the inverse of the line width of the swept source and using radio frequency shifts imprinted at each recirculation through the secondary loops, by frequency shifters.

In this paper we present a technique which allows substantial extension in the imaging range by: (i) elimination of mirror terms [14] and (ii) simultaneous acquisition of multiple axially shifted A-scans, by employing a frequency domain multiplexing method [15]. The multiple A-scans are encoded at different radiofrequencies (RF) employing the same approach as in frequency multiplexed time domain OCT [15]. The use of a multiplexer, comprising of two lenses and a pair of a Bragg cells $[16,17]$ in conjunction with a multiple delay element, allows producing several conventional A-scans at different depths simultaneously in multiple channels OCT. Each channel provides a full axial range A-scan, as it is free from mirror terms. It is also possible to adjust the differential depth difference in the multiple delay element, $\delta$, that allows superimposing of several adjacent A-scans. In this way, decreased sensitivity in an A-scan delivered by a channel is compensated by the increase in sensitivity in the adjacent channel. 


\section{Method}

\subsection{Set-up}

The schematic diagram of a long axial range SS-OCT system is depicted in Fig. 1.

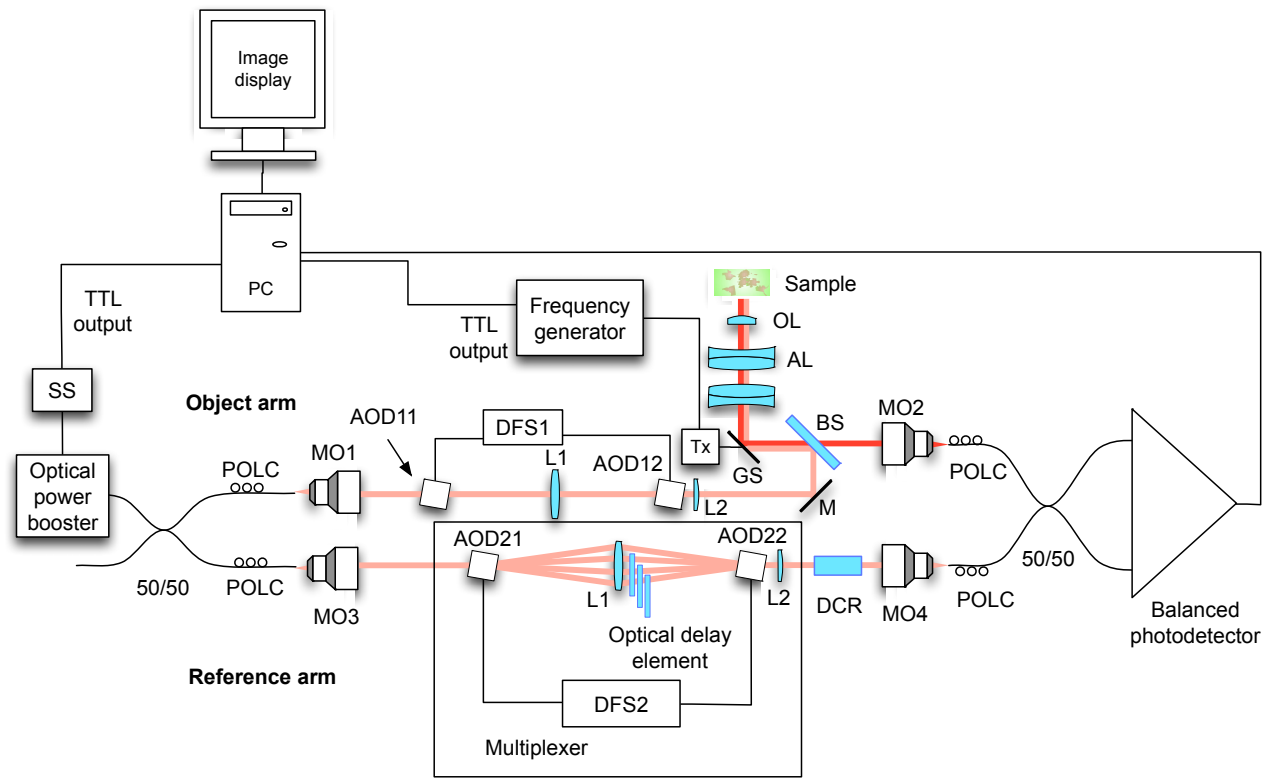

Fig. 1. Schematic layout of the long range SS-OCT system. SS: swept source; MO1,2,3,4: microscope objectives; AOD11, 12, 21, 22: acousto - optic deflectors; POLC: polarization controllers; GS: galvanometer scanner equipped with a mirror to scan along X direction; Tx: scanner controller; L1, L2: lenses; M: mirror; DFS1,2: digital frequency synthesizer; DCR: dispersion compensator rod; AL: achromatic lens; OL: Objective lens; BS: beam-splitter; PC: personal computer equipped with a NI-5124 digitizer to decode each axial scan and produce the image on the PC display.

The swept source (Superlum, Cork, Ireland, model Broad-sweeper 840) is a tunable semiconductor light source, operating at $100000 \mathrm{~nm} / \mathrm{s}$ sweeping speed, with a $828.0-873.0$ $\mathrm{nm}$ spectral range. This corresponds to scanning $\delta \lambda=45 \mathrm{~nm}$ in $0.45 \mathrm{~ms}$ at a rate $\gamma=2.2 \mathrm{kHz}$, with an equivalent repetition rate of $2 \mathrm{kHz}$. The light is amplified by an Optical Power Booster (Superlum, Cork, Ireland, model 850-50) to achieve more than $23 \mathrm{~mW}$ in single mode fiber. The light from the booster is then injected into a 50/50 single mode directional coupler that divides light into an object and a reference arm. The polarization of light is controlled using Newport F-POL-IL polarization controllers, POLC. The light output of the first coupler is collimated with two microscope objectives MO1 (New Focus 5724-B-H) and MO2 (10x OFR LLO-4-18-NIR).

The light in the reference arm then traverses two acousto-optic deflectors, AOD21 and AOD22, from Gooch and Housego. The deflectors are operated with a Gooch and Housego digital frequency synthesizer MSD040-150-0.8ADS2-A5H-8X1. The frequency synthesizer is controlled via a computer and can be tuned within $40-150 \mathrm{MHz}$ range in $1 \mathrm{kHz}$ increments. It can generate a maximum of $\mathrm{P}=8$ channels operating simultaneously with up to $200 \mathrm{~mW}$ of RF power delivered per channel. The light passing through the AOD21 can be diffracted into a number of beams, from 1 to 8 .

Lens L1 $(\mathrm{f}=500 \mathrm{~mm})$ is assembled from two closely spaced plano-convex BK7 singlet lenses, both with focal length $1 \mathrm{~m}$, and placed at $2 \mathrm{f}$ distance from the deflectors. The multiple optical delays are inserted immediately after the L1 so that each diffracted beam passes through a different glass block as depicted in Fig. 2. 
The lens L1 converges the fan of diffracted beams on the AOD22, driven by the same set of P RF signals, of identical frequencies as applied to AOD21. Light levels on each diffracted beam can be digitally controlled in each of the P channels. A lens L2 ( $\mathrm{f}=500 \mathrm{~mm})$ is placed immediately after the AOD 22 to collimate the multiplexed beam. After passing the AOD22 and L2, all beams in the first diffracted order are collimated and collinear, therefore they can be easily injected into one of the inputs of a second 50/50 single mode optical fiber coupler, using the MO4.

The object arm is equipped with the same set of Bragg cells and lenses to compensate for dispersion, however a single RF signal of $60 \mathrm{MHz}$ is applied on both AOD11 and AOD12.

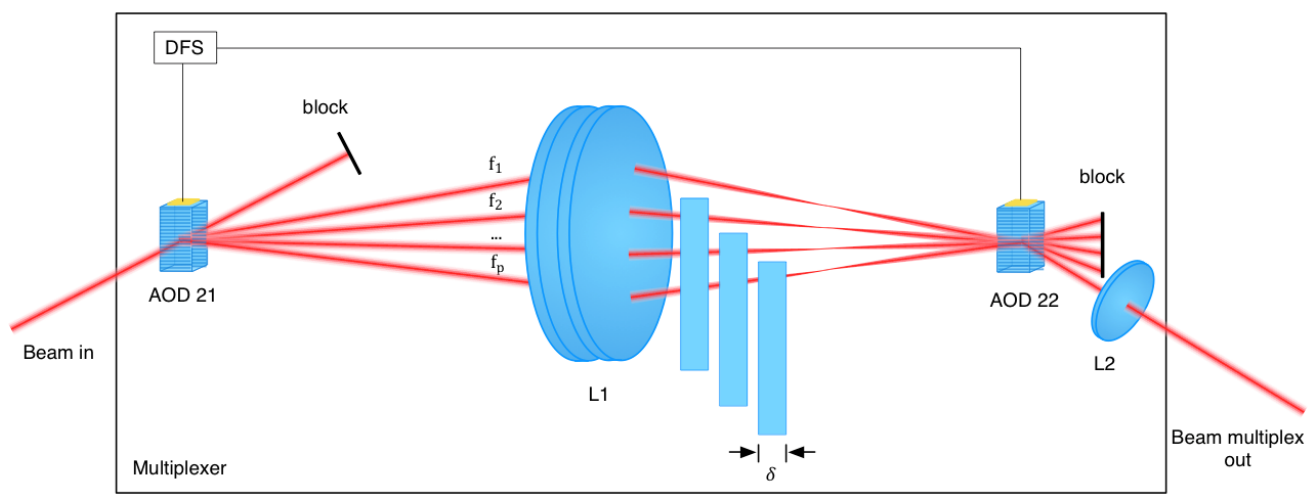

Fig. 2. Frequency division multiplexer. where each diffracted beam traverses a different delay step. The steps are made from glass plates of optical thickness $\delta$.

In the object arm, light is directed by a mirror $\mathrm{M}$ to a thick $50 / 50$ beam-splitter plate, BS, which reflects the object beam to a galvanometer X-scanner equipped with a mirror, GS. The $\mathrm{X}$-scanner determines the line in the final image and is driven by a generator $\mathrm{T}_{\mathrm{X}}$ with a sawtooth signal, generated by a Thurlby Thandar Instruments TG210 frequency generator. The TTL output from the frequency generator is connected to the PC via a NI-5124 digitizer. The beam is focused on the object using a pair of achromatic doublet lenses, AL of focal length $75 \mathrm{~mm}$, and an objective lens OL of focal length $40 \mathrm{~mm}$. The lenses are arranged in a 4f configuration, which reimages the pupil plane on the galvo-scanner mirror with magnification equal to 1 . The reflected light from the object propagates back along the same path up to the BS, wherefrom is launched via MO3 into the other input of the second 50/50 single mode fiber coupler, where it interferes with the light from the reference arm. M3 and M4 use aspheric lenses New Focus 5723-B-H. An asymmetric choice of microscope objective and lenses was made to (i) minimize the diameter of the beams travelling through the acoustooptic deflectors and (ii) increase the clearance between the beams in the reference arm at the point where the multiple delay element is inserted.

To compensate for the dispersion due to lenses AL and OL and the beam splitter BS, a dispersion compensation rod DCR is inserted in the beam after the lens L2 in the reference arm. After the AODs in both arms, only the first-order diffracted beams are selected by using pinholes placed conveniently (not shown).

The interference signal is photo-detected with a New Focus 1807 balanced photo-detector of $80 \mathrm{MHz}$ electrical bandwidth. The RF spectrum of the photodetected signal is digitized and analysed using a National Instruments PXI-5124 card, which is installed in a PC.

\subsection{Choice of excitation frequencies}

All AODs are used to simultaneously create multiple signal carriers by upshifting the frequency of each deflected beam. Sufficient separation of diffracted beams by AOD21, comparable to the size of the beam diameters of a few $\mathrm{mm}$ requires a difference of frequency in the signals applied to the AOD larger than several MHz, chosen here as $9.376 \mathrm{MHz}$. This simplifies the construction of a delay element by relaxing the step edge quality requirements 
and making the delay elements compatible with a beam diameter of up to $2 \mathrm{~mm}$. The AODs in the object arm are driven at $60 \mathrm{MHz}$ and the AODs in the reference arm are driven simultaneously at $\mathrm{f}_{\mathrm{p}}=65.247 \mathrm{MHz}+(\mathrm{p}-1) * 9.376 \mathrm{MHz}$, with $\mathrm{p}=1$ to $\mathrm{P}$. The drivers can drive up to $\mathrm{P}=8$ signals. In the experiments which follow, the maximum value for $\mathrm{P}$ was 4 . The resulting carriers in the photo-detected signal therefore pulsate at $2 \mathrm{f}_{\mathrm{p}}-120 \mathrm{MHz}$, i.e. at frequencies:

$$
C_{p}=C_{0}+(\mathrm{p}-1) \cdot \Delta \mathrm{F}
$$

where $\mathrm{C}_{0}=130.494-120=10.494 \mathrm{MHz}$ and $\Delta \mathrm{F}=18.752 \mathrm{MHz}$ as depicted in Fig. 3 [15].

\subsection{Data processing}

The data acquired by the photo-detector, is digitized by the NI PCI-5124 card, whose acquisition is triggered by the TTL sweeping signal provided by the SS and by TTL signal associated to the signal produced by the frequency generator used to drive the galvo-scanner. Thus a B-scan image is created.

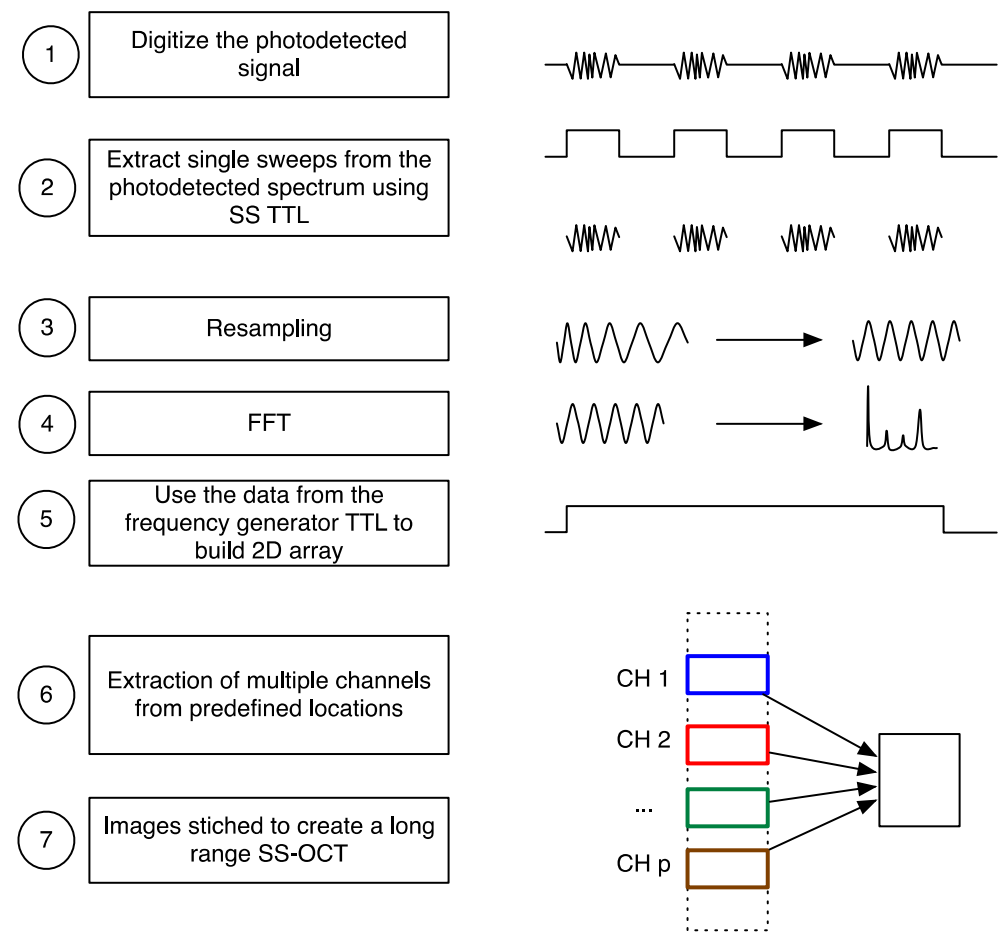

Fig. 3. Data processing algorithm.

First, the RF spectra representing single sweeps are extracted from the photo-detected signal using the TTL signal provided by the SS. The SS is driven in a saw-tooth fashion, therefore the signal due to the return of the frequency to the initial value (50 microseconds) is eliminated. Each packet extracted in this way is processed to produce an A-scan. The packets then are resampled according to known procedures in the practice of spectral domain OCT, to linearize the data, i.e. to place the data in equal frequency slots. Then a fast Fourier transform (FFT) is performed on each sweep to produce a 1D array, containing the A-scan interference signal amplitude data for each of the carriers $\mathrm{C}_{\mathrm{p}}$, as illustrated in Fig. 3. Using the data provided by the frequency generator TTL signal, multiple A-scans are assembled into a continuous A-scan. Then the A-scans are assembled into a 2D array to produce a B-scan OCT image. 
The frequency $F_{p}$ of the photo-detected signal in each channel $p$, due to sweeping the optical frequency at a rate $\gamma[\mathrm{Hz}]$, for a given value of the OPD in the interferometer is:

$$
F_{p}=\frac{\Delta k}{2 \pi} \cdot \gamma \cdot[O P D-(p-1) \delta]+C_{p}=S \cdot[O P D-(p-1) \delta]+C_{p}
$$

where $\Delta \mathrm{k}$ is the tuning bandwidth in wave numbers, $\mathrm{C}_{\mathrm{p}}$ the carrier frequency imprinted by frequency shifting on each channel $\mathrm{p}$ and $\mathrm{S}$ is the conversion efficiency of the swept source interferometer, which converts OPD into RF frequency. Using the numerical values above, $S$ $=137 \mathrm{kHz} / \mathrm{mm}$. Using Eq. (1) in Eq. (2),

$$
F_{p}=C_{0}+S \cdot O P D+(\mathrm{p}-1)[\Delta \mathrm{F}-\mathrm{S} \delta]
$$

Equation (3) shows that different channels $\mathrm{p}$ determine different frequency for the photodetected signal depending on $\mathrm{p}$.

\subsection{Choice of differential delay $\delta$}

As shown by Eq. (3), the same OPD determines different frequency components in the photodetected signal. In a different configuration applying a similar concept [13], the dependence on $p$ was eliminated by making the last bracket in Eq. (3) zero. Here however, this cannot be achieved, given the need to separate the diffracted beams out of the AOD sufficiently laterally to allow introduction of different delay elements, as explained above, which justified the choice of a step increase from one carrier to the next of $9.376 \mathrm{MHz}$. With $\mathrm{S}=137 \mathrm{kHz} / \mathrm{mm}$ and $\Delta \mathrm{F}=18.752 \mathrm{MHz}$, these would determine a value $\delta_{\mathrm{i}}$ too large for the differential step in OPD between channels of:

$$
\delta_{i}=\frac{\Delta \mathrm{F}}{\mathrm{S}}=20 \mathrm{~cm}
$$

The value of $\delta_{\mathrm{i}}$ is too large in comparison with the axial range of each channel p. Measurement of the axial range of each channel $p$, as shown below leads to a range of a few $\mathrm{mm}$. The differential delay between channels, $\delta$, should be comparable with the maximum axial range achievable per channel in order to obtain, by combination of channels, an overall constant sensitivity over an extended axial range. For instance, if a constant overall sensitivity is targeted, then sensitivity curves of neighboring channels should be shifted axially to each other until superposed over their FWHM points. This means a differential delay $\delta_{\mathrm{C}}=\mathrm{FWHM}$, where FWHM is the OPD value of the sensitivity curve versus depth where sensitivity drops to half. The measurements below will show that FWHM is approximately $2 \mathrm{~mm}$. An alternative strategy would be to accept a non-constant sensitivity with depth, but over an extended range. We adapted this strategy here and decided to shift the curves of sensitivity laterally by twice more than the FWHM of each individual channel. Three slabs of glass giving a delay $\delta=4.2 \mathrm{~mm}$ where found which satisfy this requirement. With this value in (4), the last bracket cannot be made zero (as in [11]), as $\delta<<\delta_{\text {i }}$. This means that each channel will occupy a restricted bandwidth in a spectral window around each carrier $\mathrm{C}_{\mathrm{p}}$, to a range determined by multiplying $\mathrm{S}$ with the axial range per channel, $\Delta$. Using a value $\Delta=2-4 \mathrm{~mm}$, this gives a bandwidth of plus minus $\mathrm{B} \approx 0.5-1 \mathrm{MHz}$ (an exact calculation of bandwidth is presented in the next paragraph), much less than the frequency difference between adjacent carriers, $\Delta \mathrm{F}=18.752 \mathrm{MHz}$, as shown schematically in Fig. 4(a). Figure 4(b) shows a simplified sketch of superposition of sensitivity profiles versus OPD, where $\delta \approx 2$ FWHM. This means that in order to obtain the final long range B-scan, each spectral window needs to be cropped from the photo-detected signal and shifted down in frequency by:

$$
\delta F=(\mathrm{p}-1)[\Delta \mathrm{F}-\mathrm{S} \delta]
$$

Using a differential delay of $4.2 \mathrm{~mm}$, this gives

\#185418 - \$15.00 USD Received 19 Feb 2013; revised 30 Apr 2013; accepted 30 Apr 2013; published 2 May 2013 (C) 2013 OSA 1 June 2013 | Vol. 4, No. 6 | DOI:10.1364/BOE.4.000778 | BIOMEDICAL OPTICS EXPRESS 783 


$$
\delta F=(p-1)(18.75-0.57)=(p-1) 18.18 \mathrm{MHz}
$$

In this way, the frequency generated by each channel after being shifted becomes independent on $\mathrm{p}$, giving in all cases the same value:

$$
F_{p}=C_{0}+S \cdot O P D
$$

The final long range B-scan of the curves in Fig. 4(b) is obtained by adding the intensity values of each overlapping spectral window. The frequency values in the resulting long scan can be recalculated in OPD values using an equation derived from Eq. (5):

$$
O P D=\frac{F_{p}-C_{0}}{S}
$$
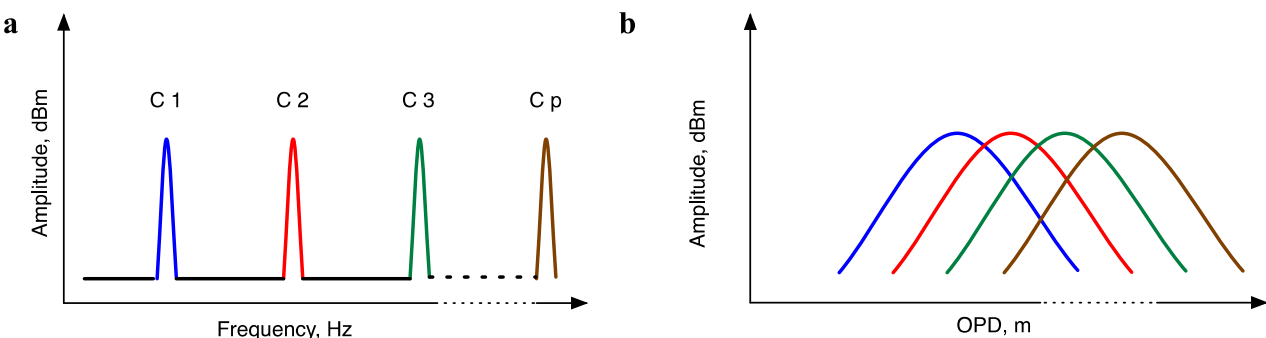

Fig. 4. a: Sensitivity curves in each channel versus optical path difference, each centered around a different carrier frequency, $\mathrm{Cp}$; b: schematic illustration of combining the sensitivity of several channels versus OPD to obtain a more constant variation of sensitivity.

\section{Results}

\subsection{Spectrum}

Figure 5 shows the sensitivity curves for 4 delayed channels and the overall cumulated curve obtained by their summation. A multiple delay element consisting in 3 steps of $\delta=4.2 \mathrm{~mm}$ and a mirror as object are used in Fig. 1. The set carrier frequencies of the four channels for $\mathrm{OPD}=0$ is $\mathrm{f}_{1}=10.494 \mathrm{MHz}, \mathrm{f}_{2}=19.87 \mathrm{MHz}, \mathrm{f}_{3}=29.246 \mathrm{MHz}$ and $\mathrm{f}_{4}=38.622 \mathrm{MHz}$. The multiple curves are raised by varying the reference arm length by moving the fiber launcher holding the MO4.

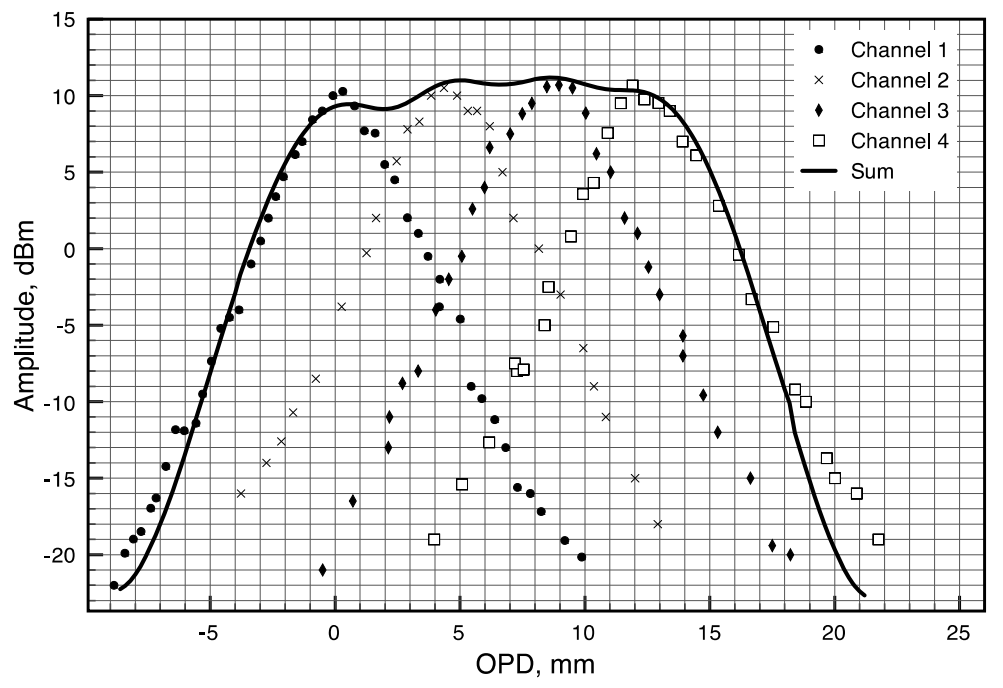

Fig. 5. Sensitivity curve for $\mathrm{P}=4$ channels delayed relatively by $\delta=4.2 \mathrm{~mm}$.

\#185418 - \$15.00 USD Received 19 Feb 2013; revised 30 Apr 2013; accepted 30 Apr 2013; published 2 May 2013 (C) 2013 OSA 1 June 2013 | Vol. 4, No. 6 | DOI:10.1364/BOE.4.000778 | BIOMEDICAL OPTICS EXPRESS 784 
The data points for each sensitivity curve were measured using a HP 8590A spectrum analyzer. The data collected were then fitted with a 4th order polynomial spline. A curve representing the long range A-Scan sensitivity was calculated as a sum of fitted curves for the single A-scan sensitivities.

Figure 5 shows that the sensitivity of each individual channel drops by more than $6 \mathrm{~dB}$ for an $\mathrm{OPD}=2 \mathrm{~mm}$ away from the maximum, giving less than $4 \mathrm{~mm}$ usable range for a single channel. However, combining the data from all 4 carriers extends the axial range of near constant sensitivity to more than $16 \mathrm{~mm}$ imaging range.

The regime of operation utilized here is of $\mathrm{P}=4$ channels with independent demodulation. The axial range is multiplied by an approximate factor of $\mathrm{P}=4$. If a swept source with 4 times longer coherence length was used, the bandwidth of the photodetector unit would have needed to accommodate a bandwidth of $4 \mathrm{~B}$, where $\mathrm{B}$ can be evaluated by considering the maximum number of axial pixels within the axial range $\delta=4.2 \mathrm{~mm}$. Using a bandwidth of $45 \mathrm{~nm}$ and a central wavelength of $0.85 \mu \mathrm{m}$, the number of pixels is $\mathrm{M}=594$. Using a scanning time of 0.5 $\mathrm{ms}$, this gives a value $\mathrm{B}=1.19 \mathrm{MHz}$. However, because here independent demodulation is implemented, the frequency of each carrier shifts from $\mathrm{Cp}-\mathrm{B}$ to $\mathrm{Cp}+\mathrm{B}$, i.e. a $2 \mathrm{~B}$ bandwidth is needed on each channel. In total, the bandwidth is not $\mathrm{P}$ times larger, but $2 \mathrm{P}$ larger, ie 2PB $=8 \mathrm{~B}$, as a disadvantage of this configuration when compared to the case of a swept source with a 4 times longer coherence length employed in a conventional single path configuration.

However, if the denser packing of carriers is employed, according to Fig. 4(b) and satisfying the Eq. (4) as in [13], then the bandwidth needed is only PB.

\subsection{Imaging}

To illustrate the capability of the system to produce a long range B-scan, in vitro imaging of a formalin fixed C57BL/J mouse tissue sample was performed. A mouse head (Fig. 6(e)) was positioned in front of OL and rotated in a way that the image would span through the major part of the sensitivity range. For this demonstration, the AODs were operated using a set of two frequencies $\mathrm{C} 1$ and $\mathrm{C} 2$. Only two channels were used for imaging because the $8 \mathrm{~mm}$ sensitivity range (where variation of sensitivity due to roll-off is less than $6 \mathrm{~dB}$ ) already exceeds the focal depth of the lens OL. 


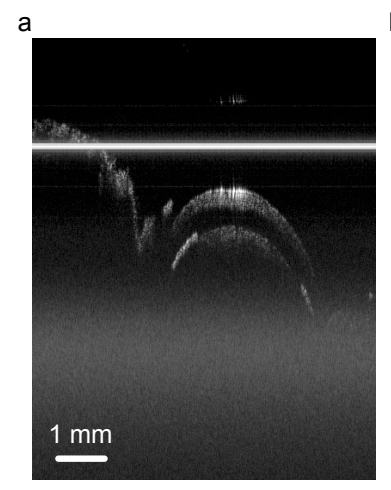

$\mathrm{CH} 1$

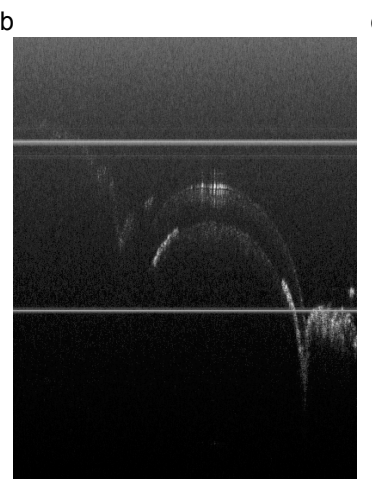

$\mathrm{CH} 2$

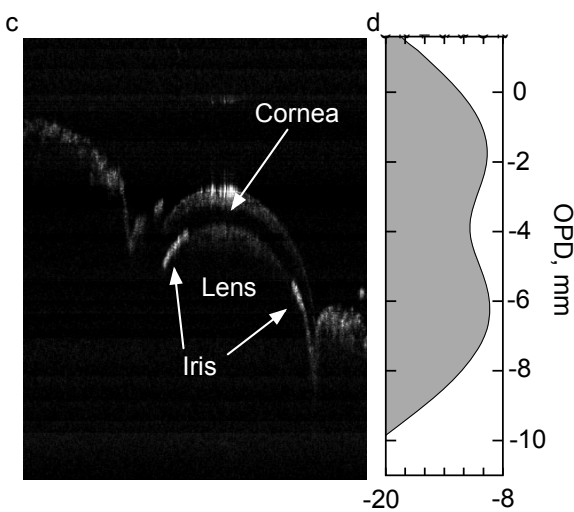

$\mathrm{CH} 1+\mathrm{CH} 2$

Amplitude, $\mathrm{dBm}$

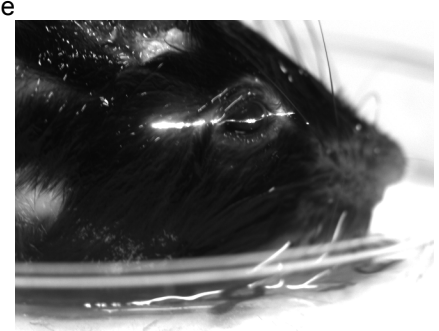

Fig. 6. B-scan images of a tilted mouse head obtained with a long-range constant sensitivity SS-OCT system. a: Conventional B-scan carried by $\mathrm{C} 1$; b: Conventional B-scan carried by $\mathrm{C} 2$; c): A long range B-scan produced by the long range imaging software; d: experimentally measured sensitivity curve in air representing the system sensitivity variation as a function of reference OPD. The curve is positioned so that OPD in the graph corresponds to the OPD in a, $\mathbf{b}$ and $\mathbf{c} ; \mathbf{e}$ a photo of the sample. The distance $\mathrm{z}$ in the object is OPD/2. Due to a long exposure time the scanning beam is visible as a white line across the eye and surrounding tissues.

Figure 6 shows two regular B-scans in Fig. 6(a) and in Fig. 6(b) that are obtained simultaneously. Each is delivered by a separate channel, $\mathrm{CH} 1$ and $\mathrm{CH} 2$ respectively, that are tuned on the carriers $\mathrm{C}_{1}$ and $\mathrm{C}_{2}$ respectively. The B-scans recorded from both channels are stitched by the imaging software to produce a long range B-scan, as presented in Fig. 6(c).

Figures 6(a) and 6(b) illustrate an additional challenge raised by this configuration. The images present several white lines due to Bragg cell intermodulation products [18]. The lines are always present at the same spectral locations when the same set of RF frequencies is applied to the Bragg cells. They are present even without interference. Therefore, these lines are removed by acquiring reference images with no object present, which subsequently are used for background subtraction from the current images before stitching them to form the final image in Fig. 6(c).

Possibly, the intensity of intermodulation peaks that are present in the RF spectrum may be significantly reduced by careful selection of acousto-optic materials and excitation RFs.

The sensitivity versus OPD curve presented in Fig. 6(d) is aligned to the images so that the graph represents the system sensitivity when acquiring the data from corresponding lines in the B-scan shown in Fig. 6(c). The position of the scanning object beam, while acquiring the long range B-scan, is visible in Fig. 6(e) as a line across the eye and surrounding tissues.

\section{Discussion}

The method implemented here is different from that reported in [13], where the differential delay between OCT channels was adjusted to match the value obtained from the difference of frequency between the channels divided by the conversion constant. S, between the OPD and the RF frequency generated when tuning the SS. Such a matching was not possible here as explained above. The method presented can be applied to up to $\mathrm{P}=8$ carriers, number limited 
here by the AOD drivers. However, in practice there should be a correlation between the compounded axial range achievable and the confocal axial range determined by the interface optics. This is why we compounded images produced by two carriers only, as the confocal depth range limited by the interface optics was below $5 \mathrm{~mm}$. Further research into long axial range OCT images should go hand in hand with methods to alleviate the limitation of sensitivity due to the confocal gate. Such methods may involve for example digital focusing [19] or use of non-conventional objective lenses [20-22].

Frequency multiplexing could be combined with recently developed emerging swept source technologies such as micro-electro-mechanical systems (MEMs) tunable vertical cavity surface emitting lasers (VCSEL) [5] to offer an even further increased axial range. Such a long axial range may find applications in sensing, profiling or tracking of objects.

The method presented is especially suitable for those swept sources where by increasing the tuning speed, their coherence length diminishes. In combining such sources with multiple path configurations as described here, to increase the axial range of the OCT system beyond the limit set by the source coherence length, the bandwidth of the photodetection unit should also be taken into account. In this paper the principle of extending the axial range was demonstrated using a configuration with $\mathrm{P}=4$ delays in 4 independent channels and using a low rate tunable swept source, where the bandwidth due to tuning was small. If the line rate is increased to the frequency of fast commercial available swept sources of $200 \mathrm{kHz}$ (100 times larger than the tuning rate used here), then a bandwidth $\mathrm{B}=119 \mathrm{MHz}$ would be needed. If non superposition of frequencies generated in the four channels is pursued, as illustrated in Fig. 4(a) and employed here, this would require values for the frequency of the first 4 carriers of at least $f_{1}=B, f_{2}=f_{1}+2 B, f_{3}=f_{2}+2 B$ and $f_{4}=f_{3}+2 B$, and so on, i.e. of at least $119,337,575$ and $813 \mathrm{MHz}$ respectively. The total photodetection bandwidth would be $2 \mathrm{~PB}=952 \mathrm{MHz}$. However, if the swept source is faster, then the adjustment used in [13] becomes achievable, in which case a more compact frequency space is achievable, as implemented in [13]. In this case, the bandwidth required is exactly that for 4 times more axial pixels, i.e. $476 \mathrm{MHz}$ only.

Swept sources with tuning frequencies of over $1 \mathrm{MHz}$ [23] have also been reported, which would require acousto-optic modulators and photodetecting units operating at frequencies over several GHz.

In our experiment, the setup presented was optimized to offer a long sensitivity range on the expense of some allowed variation of sensitivity within the range. The ripples in the overall sensitivity curve are less than $2 \mathrm{~dB}$. Reduced rippling can be obtained by choosing a differential delay element $\delta$ so that the imaging channels would come even closer together, in terms of OPD, providing a relatively flat top sensitivity curve when combined, as suggested by the profile in Fig. 4(b).

\section{Conclusions}

A system capable to perform long range SS-OCT imaging by combining several mirror terms free B-scans acquired simultaneously at different depths is presented. The system uses AOM frequency shifters implementing a multiple delay element with $\mathrm{P}$ delays in the reference arm, each traversed by a deflected beam at a different angle and with a different frequency shift as imprinted by the AOMs. The part of the reference beam traversing a particular delay in the multiple delay element is coded in the RF frequency of the photo-detected signal. In this way, A-scans and B-scans corresponding to different axial ranges can be identified.

The system is demonstrated for $\mathrm{P}=4$ delays which covers more than $17 \mathrm{~mm}$ OPD axial range measured at $6 \mathrm{~dB}$, with a variation of less than $2 \mathrm{~dB}$ inside this axial range. The capability of producing long-range B-scan images was proven by imaging a part of a mouse head extended over $1 \mathrm{~cm}$. The method presented acts on the interferometer configuration and not on the source and therefore can be combined with any method acting on the swept source.

\section{Acknowledgments}

The research leading to these results has received funding from the European Research Council under the European Union's Seventh Framework Programme, Advanced Grant 
agreement 'COGATIMABIO', No: 249889. A. Podoleanu is also supported by the NIHR Biomedical Research Centre at Moorfields Eye Hospital NHS Foundation Trust and UCL Institute of Ophthalmology. The authors also acknowledge Philip J. Broadbridge for preparing the mouse head sample.

\#185418 - \$15.00 USD Received 19 Feb 2013; revised 30 Apr 2013; accepted 30 Apr 2013; published 2 May 2013 (C) 2013 OSA 1 June 2013 | Vol. 4, No. 6 | DOI:10.1364/BOE.4.000778 | BIOMEDICAL OPTICS EXPRESS 788 